\title{
Incentive reduction: Varied simultaneous reductions and subsequent responding'
}

JAMES H. McHOSE, DEPARTMENT OF PSYCHOLOGY, SOUTHERN ILLINOIS UNIVERSITY, Carbondale, Ill. 62901

Delay and magnitude of reinforcement in the first goal box $\left(G_{1}\right)$ of a double alley apparatus were manipulated in two experiments. The results indicated that a decrease in reward magnitude in $G_{1}$ accompanied by an increase in delay of reinforcement elevated the speed with which rats traversed the second alley $\left(A_{2}\right)$. Facilitation of $A_{2}$ speeds was positively related to the length of delay increase, but inversely related to the amount of reward reduction.

Previous data indicate that certain incentive reductions (IRs) in the first goal box $\left(G_{1}\right)$ of the double alley apparatus elevate speeds in the second alley $\left(A_{2}\right)$ as compared with those of a (control) group regularly receiving the reduced incentive value in $G_{1}$. The class of IRs which facilitate subsequent responding would appear to include those in which reward magnitude is decreased simultaneous with an increase in delay of reinforcement. Thus, nonreinforcement of a previously reinforced response, which may be viewed as an end point on delay and magnitude change continuua, has been shown to facilitate subsequent responding (Barrett et al, 1965; Wagner, 1959). Incomplete reduction in reward magnitude, to some nonzero magnitude, facilitates subsequent responding when accompanied by an increase in delay of reinforcement (McHose, 1966b), although neither incomplete reward reduction nor delay increase alone are sufficient to produce such facilitation (Barrett et al, 1965; McHose, 1966a).

The present paper reports two experiments designed to determine whether variations in magnitude reduction and delay increase within simultaneous magnitude reduction-delay increase manipulations produce differential facilitation of $\mathrm{A}_{2}$ speeds.

Method. In Experiment 1, 64 male albino rats received 48 acquisition and 32 postshift trials in an L-shaped double alley apparatus previously described (McHose, 1966a). Briefly, the apparatus consisted of a first start, run and goal section, and a second run and goal section. The first goal box $\left(G_{1}\right)$ served as the start box for the second alley $\left(A_{2}\right)$.

Ten days prior to the first training day Ss were placed on a $23 \mathrm{~h}$ food deprivation cycle maintained throughout the experiment. On the two days immediately preceding the first training day Ss received approximately $1 \mathrm{gm}$ of $45 \mathrm{mg}$ Noyes pellets, identical with the subsequent reinforcement pellet, prior to their daily ration. Ss were also allowed to explore $S_{1}$ and $A_{1}$ on these days. Each $S$ received two trials on each of the first two training days and four trials per day for the remainder of the experiment.

Three randomly constituted groups were designated according to the reinforcement condition (no. pellets-delay in sec) received in $G_{1}$ on each acquisition trial: Groups $8-0(n=22), 3-0(n=20)$, and $1-30(n=22)$. Postshift conditions remained the same except that Groups $8-0$ and $3-0$ received two test trials of 1 pellet at $30 \mathrm{sec}$ delay (1-30) in each daily block of four trials according to the following repeating cycle of test $(\mathrm{T})$ and standard (S), or acquisition value, conditions: STST, TSST, TSTS, STTS. All Ss always received two pellets immediate reinforcement in the second goal box $\left(G_{2}\right)$.

In Experiment 2, 29 male albino rats received 54 acquisition and 45 postshift trials in the apparatus of Experiment 1. Deprivation and prefeeding procedures were identical to those of Experiment 1. Each $S$ received one trial on the first day of training, two trials on the second day, and three trials per day for the remainder of the experiment.

Three groups were designated according to the reinforcement condition (no. pellets-sec delay) received in $G_{1}$ on each acquisition trial: Groups $5-0$ (n $=9), 5-15(n=10)$, and 1-30 $(n=10)$. During the postshift period Groups 5-0 and 5-15 received one pellet at 30 sec delay $(1-30)$ in $G_{1}$ on one of their three daily trials and their respective acquisition reinforcement condition on the remaining two trials of each daily block of trials. Group 1-30 continued to receive the 1-30 reinforcement condition all postshift trials. All Ss always received two pellets immediate reinforcement in $G_{2}$.

In both experiments the delay of reinforcement cycle began when $S$ interrupted a photobeam located 1 in. in front of the goal cup in $G_{1}$, the start door separating $G_{1}$ from $A_{2}$ was opened after $S$ had eaten and maintained an orientation toward the door for $3 \mathrm{sec}$, and reciprocal traversal times over a 1 -ft section of $A_{2}$, beginning 6 in. from $G_{1}$, were recorded.

Results. Group mean speeds over the postshift period of Experiment 1 are shown in Fig. 1. Mean speeds on 8-0 and 1-30 trials for Group 8-0, and 3-0 and 1-30 trials for Group 3-0, are plotted separately for each block of eight trials. As may be seen in Fig. 1, reduction in reward to 1-30 decreased the speeds of Group 3-0. Analysis of variance indicated that this decrease in speeds within Group 3-0 was statistically significant $(\mathrm{p}<.001)$ over trials $65-80$ (Blocks $3-4)(F=21.80$, $\mathrm{df}=1 / 19)$. Of primary interest, however, is the observation that Group 3-0 speeds on 1-30 trials were faster than those of either Groups $1-30$ or $8-0$ on 1-30 trials. Variance analysis of the data for the three groups on 1-30 trials over Trials 65-80 (Blocks 3-4) yielded a significant $(\mathrm{p}<.01)$ groups effect $(F=5.49, \mathrm{df}=2 / 61)$. A subsequent $t$ test indicated that speeds for Groups 8-0 and 1-30 did not significantly differ on these trials.

Finally, as may be seen in Fig. 1, three pellet immediate reward (Condition 3-0) resulted in faster speeds than did eight pellet immediate reward (Condition 8-0) even though these conditions involved no change in reward, for Groups 3-0 and 8-0 respectively, from the pre- to the postshift phase of the experiment. Variance analysis of the data represented by Conditions $3-0$ and $8-0$ over Blocks 3-4 yielded a significant $(\mathrm{p}<.001)$ groups effect $(\mathrm{F}=$ 30.71 , df $=1 / 40$ ).

Group mean running speeds over the postshift period of Experiment 2 are also shown in Fig. 1. Mean speeds on 5-0 and 1-30 trials for Group 5-0, and on 5-15 and 1-30 trials for Group 5-15, are plotted separately for each block of nine trials. Of primary interest is the observation that Group 5-0 speeds were faster on 1-30 trials than those of either Group 1-30 or Group 5-15 on 1-30 trials. Variance analysis of speeds on 1-30 trials over Trials 73-99 (Blocks 3-5) yielded a significant $(p<.01)$ groups effect $(F=6.27, d f=2 / 25)$. Mean speeds of Groups 1-30 and 5-15 (1-30) did not significantly differ over Blocks 3-5. Of secondary interest is the observation that Group 5-0 speeds on 1-30 trials were slightly above those on 5-0 trials. This difference, over Blocks 3-5 was significant $(F=5.67, \mathrm{df}=1 / 8, \mathrm{p}<.05)$.

Finally, analysis of the data represented by Conditions $5-0$ and 5-15 over Blocks 3-5 yielded a significant $(p<.025)$ groups effect $(\mathrm{F}=7.97, \mathrm{~d} f=1 / 17)$.

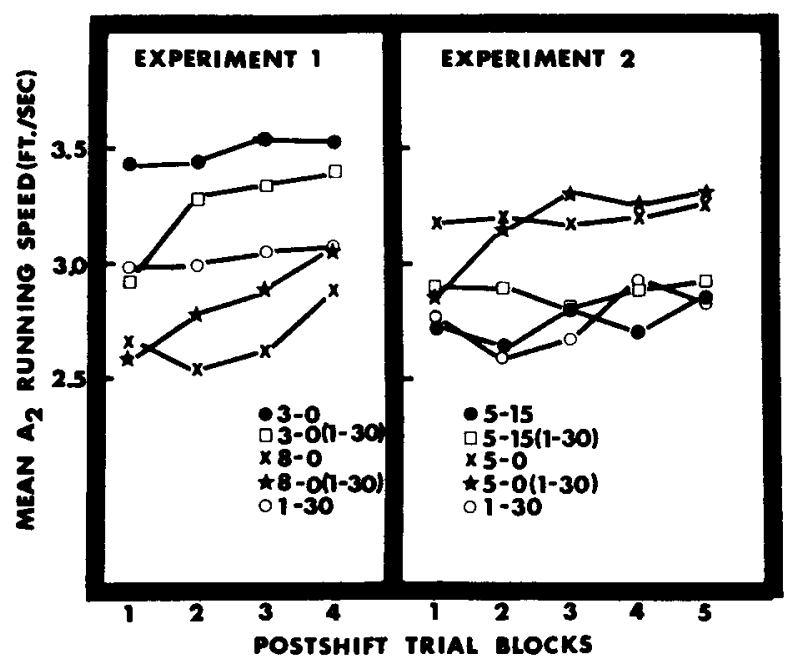

Fig. 1. Mean $A_{2}$ running speeds as a function of trials for the various reinforcement conditions of Experiments 1 and 2 . 
Discussion. The present data, in accord with previous findings (McHose, 1966b), indicate that a decrease in reward magnitude accompanied by an increase in delay of reinforcement in $G_{1}$ enhances subsequent $\left(A_{2}\right)$ responding. Thus $A_{2}$ speeds of Groups $3-0$ (Experiment 1 ) and $5-0$ (Experiment 2) on 1-30 test trials were above those of their respective 1-30 (control) groups. Moreover, the facilitative effect of such simultaneous IRs was found to vary with both the reward magnitude and delay components of the incentive reduction. The amount, or occurrence, of facilitation on simultaneous IR test trials varies directly with the amount of delay increase (Experiment 2), but inversely with the amount of reduction in reward magnitude (Experiment 1).

While variation in the delay increase component of simultaneous IRs currently has no analog in the literature concerned with facilitative effects of nonreward, variation in the reward reduction component of simultaneous but incomplete IRs is analogous to nonreinforcement following training under different magnitude of reward conditions between groups. The observation, in Experiment 1, that the effect of an increase in reward reduction was to decrease the amount of facilitation is consistent with the finding that the facilitation of $\mathbf{A}_{2}$ speeds following nonreward varies inversely with the magnitude of reward regularly received in $G_{1}$ (Barrett et al, 1965). It should be noted, however, that the latter finding is by no means ubiquitous (cf Krippner et al, 1967; McHose \& Ludvigson, 1965).

The present data constitute a portion of a substantive literature which indicates that certain IRs augment the vigor of responses occurring immediately subsequent to the reduction. They further suggest that the facilitative properties of reduction in incentive vary with the parameters of the reduction. The observation that the IRs of the present study facilitate subsequent responding may be interpreted within the context of the expectancy-frustration-drive model developed by Amsel (1958). The finding that the amount of response facilitation attending an IR varies inversely with the amount of reward reduction, however, is seemingly inconsistent with one of the basic assumptions of frustration theory, that the energizing effect of an IR is positively related to reward expectancy.

\section{REFERENCES}

AMSEL, A. The role of frustrative nonreward in noncontinuous reward situations. Psychol. Bull, 1958, 55, 102-119.

BARRETT, R. J., PEYSER, C. S., \& McHOSE, J. H. Effects of complete and incomplete reward reduction on a subsequent response. Psychon. Sci. $1965,3,277-278$.

KRIPPNER, R. A., ENDSLEY, R. C., \& TACKER, R. S. Magnitude of $\mathrm{G}_{1}$ reward and the frustration effect in a between subjects design. Psychon. Sci, 1967, 9, 385-386.

McHOSE, J. H. Incentive reduction: Delay increase and subsequent responding. Psychon. Sci, 1966a, 5, 213-214.

McHOSE, J. H. Incentive reduction: Simultaneous delay increase and magnitude reduction and subsequent responding. Psychon. Sci., 1966b, $5,215-216$.

McHOSE, J. H., \& LUDVIGSON, H. W. Role of reward magnitude and incomplete reduction of reward magnitude in the frustration effect. $J$. exp. Psychol, 1965, 70, 490-495.

WAGNER, A. R. The role of reinforcement and nonreinforcement in an "apparent frustration effect." J. exp. Psychol, 1959, 57, 130-136. NOTE

1. Supported by Grant MH 10340 from the United States Public Health Service. 